\title{
Reply to Gregory Landini's Review of Formal Ontology and Conceptual Realism
}

\author{
Nino B. Cocchiarella
}

\section{Some Initial Ontological Distinctions}

In our discussion of Greg Landini's review, we should distinguish how the logical systems $\lambda H S T^{*}$ and $H S T_{\lambda}^{*}$ that I have developed are to be understood in my reconstructions of Gottlob Frege's and Bertrand Russell's (1903) ontologies as opposed to how $H S T_{\lambda}^{*}$ is understood as a (proper) part of my ontology of conceptual realism. Both of these systems are type-free second-order predicate logics that allow predicate expressions (complex or simple) and formulas (propositional forms) to be nominalized and occur in formulas as abstract singular terms. ${ }^{1}$

The main logical difference berween these systems, as Landini notes, is that whereas $\lambda H S T^{*}$ contains standard first-order logic (with identity) as a proper part, the system $H S T_{\lambda}^{*}$ is free of existential presuppositions regarding singular terms, including nominalized predicates as abstract singular terms, which is essential to any argument for Russell's paradox of predication. In particular, nominalization of the Russell predicate that is not predicable of itself turns out to be denotationless in $H S T_{\lambda}^{*}$ as an abstract singular term.

The main ontological difference between Russell's and Frege's ontologies is that one is intensional and the other is extensional. Russell's (1903) ontology is based on predication as the ontological nexus of propositions, whereas Frege's is based on predication as a function from properties and relations to truth values. $^{2}$ In conceptual realism, predication is based on the mutual saturation of referential and predicable concepts as unsaturated complementary cognitive structures, the result being a speech or mental act. ${ }^{3}$

In Russell's ontology, a nominalized predicate denotes, as an abstract singular term, the very same property or relation (in-intension) that the predicate stands for in its role as a predicate. In Frege's ontology, a nominalized predicate denotes the extension (value range, Wertverläufe) of the concept or relation (qua

\footnotetext{
${ }^{1}$ There is of course a type distinction between object terms and predicates in these systems; but unlike the situation in type theory there is no hierarchy of predicates of different types.

${ }^{2}$ Frege's Begriffe are really Eigenshaften, and in our in present context where we want to distinguish concepts as cognitive capacities from Frege's Begriffe, it is better to speak of his Begriffe as properties instead.

${ }^{3}$ We assume in this discussion a distinction between predication in language, predication in our speech and mental acts, and predication as the nexus of propositions or of states of affairs, or, in Frege's case, as functional application.
} 
function from objects to truth values) that the predicate stands for in predicate position; and in conceptual realism, a nominalized predicate denotes the intension of the concept ( qua cognitive structure) that the predicate stands for in its role as a predicate. Because what a predicate stands for and what its nominalization denotes are not the same type of entity in either Frege's ontology or my conceptual realism, the fact that a nominalized predicate, on pain of contradiction, might fail to denote as an abstract singular term does not affect the objective reality of what that predicate stands for in its role as a predicate. That is why the system $H S T_{\lambda}^{*}$ can be used in a reconstruction of Frege's ontology as well as in my conceptual realism. The system $\lambda H S T^{*}$ will also suffice for a reconstruction of Frege's ontology, but a free first-order predicate logic is essential to my analysis of plurals and mass nouns in terms of the logic of classes as many developed in my book, which means that only $H S T_{\lambda}^{*}$ is appropriate for conceptual realism.

On the other hand, for a reconstruction of Russell's ontology, where nominalized predicates denote the same property or relation they stand for in their role as predicates, only the system $\lambda H S T^{*}$ is appropriate. That is, because it is the same entity involved in both roles in Russell's ontology, we cannot in that framework both affirm the being of what a predicate stands for in its role as a predicate, and also deny that being in the nominalization of the predicate as an abstract singular term.

\section{Russell's Paradox of the Totality of Propositions}

Landini claims that Russell's paradox of the totality of propositions is a paradox about hyper-intensionality. I disagree. There is nothing in Russell's discussion or argument for this paradox in Appendix B of the 1903 Principles of Mathematics that suggests that any hyper-intensionality principles are involved or assumed in that argument. Indeed the logical context for that discussion and argument was Russell's initial version of a simple theory of types (sans any hyper-intensionality principle), which as I have shown is equivalent to both $\lambda H S T^{*}$ and $H S T_{\lambda}^{*}$. And then, from the time of Principia Mathematica in 1910-13 and thereafter, Russell rejected propositions as real objective truths or falsehood, which suggests that hyper-intensionality was also not involved in Russell's ramified theory of types.

But Landini is in good company. In 1974, Alonzo Church suggested modifying the type-theoretical language of simple type theory by adding a new primitive sign for propositional identity along with certain new axioms that amount to what others have come to call a hyper-intensional logic. Later, in 1984, Church showed how Russell's paradox of the totality of propositions can be resolved in this hyper-intensional logic. Since then others have tried to see if they could construct new paradoxes using principles of hyper-intensionality. Whatever one thinks of this later development, it is important that we separate the original context of Russell's 1903 ontology (or my conceptual realist ontology) from the ontology of a later assumption of hyper-intensional principles, type-theoretical or otherwise. It is clear that Church's different resolution of the paradox does not mean that my resolution is somehow defective. In other 
words, hyper-intensional principles in Church's theory of types is one way of resolving the paradox of the totality of propositions, but my way for the typefree system $\lambda H S T^{*}$ (and $H S T_{\lambda}^{*}$ ) shows that we do not need hyper-intensional principles to solve the paradox.

My resolution of Russell's paradox of propositions in the context of $\lambda H S T^{*}$ is correct, in other words, regardless whether or not we think that there might be yet another paradox if we are inclined to add principles of hyper-intensionality to $\lambda H S T^{*}$. Certainly, as I have explained in my book, Serge Bozon's argument that "there is a property-theoretic variant of this paradox" does not apply to either Russell's, Frege's or my conceptual realist ontology as reconstructed by $\lambda H S T^{*}$ or $H S T_{\lambda}^{*}{ }^{4}$

Landini is not entirely pleased with certain aspects of my solution, however. In particular, he objects to my way of representing the nominalization of a propositional form (formula) $\varphi$ as $[\lambda \varphi]$, which is read as 'that $\varphi$ ' (as in 'that snow is white'). Now according to clause 4 of the rules of my logical syntax for $\lambda H S T^{*}$ the expression $[\lambda \varphi]$ is generated as a 0 -place predicate expression, which as a predicate can be nominalized and treated as an abstract singular term. That, of course, is the whole point of introducing such an expression. ${ }^{5}$ But, by clause 3 , an $n$-place predicate applied to $n$ terms results in a formula, for all natural numbers $n$, including 0 , from which it follows that $[\lambda \varphi]$ is a formula as well.

Landini says that with "0-place predicate terms added to the language, a predicate is permitted in the position of a formula flanking a logical particle" such as the conjunction sign. This is true for propositional variables, which as 0place predicate variables are simple formulas (by clause 1 of my logical syntax); and it is true for $[\lambda \varphi]$ as well. This may seem odd to some, because syntax categories are usually exclusive of one another; but there's nothing inconsistent about it.

Why have 0-place predicate expressions at all? Well, this goes back to the work of Richard Montague in his development of a type-theoretical intensional logic (sans principles of hyper-intensionality). Montague was my teacher and mentor in this area, and I thought this was a nice simplifying way of looking at propositional variables. But I have no objection to dropping the terminologyand that is all that is really involved here - and simply call 0 -place predicate variables propositional variables instead.

As far as taking $[\lambda \varphi]$ as a 0 -place predicate expression, and hence a formula as well, even I find this awkward and prefer simply writing $[\varphi]$ as an abstract singular term (but only as such) instead. It is of course a trivial matter to change the rules of the logical syntax for $\lambda H S T^{*}$ to exclude $[\lambda \varphi]$ as well-formed altogether, and just introduce $[\varphi]$ as an abstract singular term. In the end it's a matter of how natural one finds the syntax. I find nothing substantive in this

\footnotetext{
${ }^{4}$ Bozon 2004, p.1.

${ }^{5}$ In the definition of my logical syntax for $\lambda H S T^{*}$ on page 96 of Cocchiarella 2008, I add a footnote stipulating that if $n=0$, then we are to take $a_{1}, \ldots, a_{n}$ as the empty sequence. It is this stipulation that allows $[\lambda \varphi]$ to be a 0 -place predicate expression by clause 4 . We need only require that $n \geqslant 1$ to exclude this possibility.
} 
issue.

\section{Hyper-intensionality in Russell's Early Ontology}

Hyper-intensionality principles were designed by Church to reflect a finergrained analysis of the structure of propositions - which he assumed to be complex abstract entities - than could be represented by mere logical equivalence. Although the proposition expressed by $(P \vee(Q \wedge \neg Q))$, for example, is logically equivalent to (and therefore has the same truth conditions as) the proposition expressed by $P$ alone, nevertheless, according to Church, they do not express the same proposition. The addition of hyper-intensional principles to simple type theory was designed to capture the identity conditions of propositions as complex abstract structures. And in his 1903 ontology, Russell was in fact committed to propositions as complex abstract entities (but then after 1910 he rejected propositions as abstract entities).

As noted in my book, what Russell meant (in 1903) by an ontological complex was an object whose "being presupposes the being of certain other terms [objects]". ${ }^{6}$ He recognized only two kinds of complexes at that time, namely, classes as ones (sets), whose being presuppose the being of their members, and propositions. Of course, classes as ones are extensional entities, and hyperintensionality principles do not apply to them. Nothing at all was said about the complexity of properties and relations. Propositional functions were nonentities in 1903, and later they became complex expressions that were associated with properties and relations.

As independently real, abstract entities of Russell's 1903 ontology, in other words, some properties and relations are represented by complex predicate expressions (or what Russell called propositional functions); and that in fact is exactly the function of the comprehension principle of $\lambda H S T^{*}$, which is formalized as:

$$
(\exists F)\left(F=\left[\lambda x_{1} \ldots x_{n} \varphi\right]\right),
$$

where the $\lambda$-abstract is homogeneously stratified (in the metalanguage). This principle compares nicely with the first-order formula $(\exists x)(x=a)$ as a way to indicate that the object expression $a$ denotes (a value of the bound object variables). But, just as the complexity of the object term $a$ (as in the case of definite descriptions or applied functors) does not indicate a corresponding ontological complexity in what $a$ denotes, so too the above comprehension principle does not mean that the property or relation that the $\lambda$-abstract stands for is an ontologically complex entity that has other properties and logical relations as constituents. Rather, what it means is that there are logical relations that hold between the properties and relations as represented by such a complex expression and those represented by the constituent predicates that occur in that expression. It is this view of properties and relations that is represented in $\lambda H S T^{*}$, as Landini notes, and not that of an ontology of complexes.

Now, the arguments for a paradox about hyper-intensionality depend on the assumption that complex predicates represent complex properties and relations,

${ }^{6}$ Russell 1903, §133, p. 137. 
which is not true in Russell's or Frege's ontology, or in conceptual realism. But even given such an assumption, there are problems with the hyper-intensional principles assumed by Bozon. I will not repeat my criticism of these principles here, however, and certainly Landini's discussion of this paradox should suffice to see what has gone wrong with Bozon's argument. And, in regard to the simpler version of the paradox formulated by Landini himself in his review, this is resolved, as he says, by requiring that the $\lambda$-abstract $[\lambda z \varphi]$, where $z$ does not occur free in $\varphi$, should be typed the same way as $[\lambda \varphi]$. I agree that this is the appropriate step to take.

Landini makes a rather novel proposal regarding how to avoid assuming hyper-intensional principles if we think that propositions are complex entities to which some such principles should apply. His proposal is that we should drop the nominalized propositional form $[\varphi]$ corresponding to each formula $\varphi$, and instead use a vacuously bound $\lambda$-abstract $[\lambda z \varphi]$, where $z$ is not free in $\varphi$, as a 1 -place predicate expression. This is not a "vacuous" attribute if $\varphi$ is true, it should be noted, because in that case the predicate is true of everything. It is vacuous, in other words, if, and only if, $\varphi$ is false. The idea is that if nominalized propositional forms are eliminated, then we need no longer worry about hyperintensionality, at least not in Russell's early framework once propositions as abstract entities are dropped from the ontology (as in fact Russell later did).

I do not think this proposal will work for conceptual realism, however. We need a more perspicuous way to represent the cognitive structure of our speech and mental acts in which a nominalized sentence will occur when we use a predicate for an intentional verb such as 'know', 'believe', 'think', etc.. We also need to develop a logic of these intentional verbs within the general framework of conceptual realism, and in particular a logic that is based upon a finer-grained analysis of the structure of nominalized sentences than I have given so far.

Of course, if a logic of intentional verbs can be developed on the basis of Landini's proposal, that might be a preferable way to go. In that regard I have no real objection to that approach and wish success in constructing such a logic to Landini and others who prefer his option instead.

\section{Hyper-intensionality in Conceptual Realism}

Predicable concepts in conceptual realism are unsaturated cognitive capacities that underlie our rule-following abilities in the use of predicate expressions; and as such they determine the correct use and therefore truth conditions of those predicates in different contexts of use. As a cognitive capacity, the concept that a complex predicate expression stands for is not itself a complex of the concepts and logical relations that the component parts of that expression stand for. That is why hyper-intensionality principles do not apply to the predicate expressions of conceptual realism in their role as predicates, and in particular to those that are used in our speech and mental acts. In this regard, Landini is correct in noting that I reject hyper-intensional principles as applied to referential and predicable concepts.

Intensional objects, as the objectified contents of concepts do not have an unsaturated nature, on the other hand, and as I suggested in my book, we might 
very well want to view them as complexly structured objects subject to some hyper-intensional principles. The problem is how are these complexly structured objects to be semantically characterized and what hyper-intensional principles do they validate.

One approach might be to first semantically distinguish the quasi-intensions of expressions, i.e., the functions from contexts of use to extensions of the relevant sort, that are determined by our concepts as cognitive capacities. ${ }^{7}$ Then, assuming quasi-intensions, we go on to distinguish the intensions of those expressions in a stricter sense, and in particular a sense that represents their fine-grained structure as well as the quasi-intensions of their component parts. ${ }^{8}$ The question is: how are we to semantically characterize these intensions in a stricter sense?

One proposal might be to identify the intensional object denoted by a nominalized sentence (or complex predicate expression) with a well-ordered sequence of the quasi-intensions denoted by the component parts of that sentence (including that of the sentence, or complex predicate, itself), i.e., a sequence ordered in accordance with the way that the sentence (or complex predicate) is inductively constructed. Of course all of the predicate and referential expressions occurring in a nominalized sentence, or in a nominalized complex predicate, are understood to be deactivated.

Some hyper-intensional principles will be validated under such an analysis. The sequence denoted by a nominalized conjunction $(\varphi \wedge \psi)$, for example, will be different from the sequence denoted by the nominalization of the logically equivalent sentence $(\psi \wedge \varphi)$, so that the two nominalizations will denote different intensional objects. In this way the hyper-intensional thesis:

$$
\left[P_{1} \wedge Q_{1}\right]=\left[P_{2} \wedge P_{3}\right] \rightarrow P_{1}=P_{2} \wedge Q_{1}=Q_{2}
$$

will be validated. Of course, there will be similar theses validated for disjunction, conditionals and biconditionals; and then there will be still other such theses as well. Determination of which hyper-intensional principles are valid and whether a consistent set of such principles can be added to $\lambda H S T^{*}$ or $H S T_{\lambda}^{*}$, and whether a completeness theorem could then be proven for these principles are issues that must be left to future research.

One might object to this proposal as being an unnatural view of the strict intensional content of a sentence; but I suspect that such an objection assumes that such propositional content is somehow "present before the mind" in such a way that the structure of that content is part of the "presentation". Nevertheless, even granting that, it might very well be that having such a "presentation" before the mind involves a comprehension of the inductive steps involved in the construction of that content.

\footnotetext{
${ }^{7}$ Quasi-intensions, as functions from contexts of use (or possible worlds), are what are usually called intensions in modal logic. The quasi-intension of an $n$-ary predicate, e.g., is a function from possible contexts of use to classes of $n$-tuples in the domains of those contexts. The quasi-intension of a sentence is the function from contexts of use to the truth values, truth or falsehood.

${ }^{8}$ Intensions in a stricter sense are usually called senses.
} 
How well this proposal can be consistently carried out is, as I have said, a project for future research. There might of course be other ways to interpret intensional objects so that the structure of a formula (or complex predicate) will be a determinant in how intensional objects are individuated. The main point to note here is that hyper-intensional principles can be applied in conceptual realism, but only to intensional objects and not at all to referential and predicable concepts, and therefore not at all to referential and predicate expressions in their primary roles.

\section{Frege's Hierarchy of Levels of Concepts}

Landini suggests a different approach to representing complex intensional objects, and in particular one that does not assume any hyper-intensional principles. Landini would utilize Frege's higher levels of concepts instead, but with concepts understood as in conceptual realism (i.e., as cognitive structures) as opposed to Frege's own view of concepts as abstract functions.

Actually, in his review, Landini deals only with the first three levels of Frege's hierarchy: namely, the initial level of objects, the first-level concepts, and the second-level of referential-quantifier concepts. (I am not sure how going any higher is relevant to Landini's proposal.) What Landini describes is an extension of $\lambda H S T^{*}$ (and $H S T_{\lambda}^{*}$ as well I assume) in which quantifiable variables for the (unsaturated) quantifier phrases of Frege's second level are added.

I described such an extension of these systems in a 1992 paper and noted that (in the monadic case)

$$
(\forall \mathcal{Q})(\exists F)(F=[\lambda y(\exists G)(y=G \wedge(\mathcal{Q} x) G(x))])
$$

is an instance of the comprehension principle of this extension of $\lambda H S T^{*}{ }^{9}{ }^{\mathrm{I}}$ then noted that Frege's double-correlation thesis follows; that is,

$$
(\forall \mathcal{Q})(\exists F)(\forall y)(F(y) \leftrightarrow(\exists G)[y=G \wedge(\mathcal{Q} x) G(x)])
$$

is then provable. What this thesis says is that for every second-level (referentialquantifier) concept $\mathcal{Q}$, there is a first-level concept $F$ such that all and only the concepts that fall within $\mathcal{Q}$ fall under $F$. "The provability of this thesis," in such an extension or otherwise, I then noted, "indicates the superfluousness of extending the system $\lambda H S T^{*}$ through the addition of quantifiable variables for variable-binding operators, i.e., for variables that range over second-level concepts." 10 The question here is: given the double-correlation thesis do we really need to extend these systems in the way that Landini proposes; that is, is such an extension not superfluous after all?

The example Landini considers is the sentence ' $S$ believes that the author of Waverly is brilliant'. As a speech act by someone (other than $S$ ) this sentence is initially symbolized in my framework as

$$
S \text { Believes }\left[\left(\exists_{1} x W\right) \text { Brilliant }(x)\right],
$$

\footnotetext{
${ }^{9}$ Cocchiarella 1992.

${ }^{10}$ Ibid., p. 189 f.
} 
where $\left(\exists_{1} x W\right)$ is read as 'the author of Waverly', and where the symbolization of the sentence 'The author of Waverly is brilliant' has been nominalized (and placed in brackets to indicate this). ${ }^{11}$

First, let us note that as a speech act this sentence might have either a de re reading or a de dicto reading. Landini considers only the de re reading, apparently because this is the one that on his account shows how to avoid the paradoxes he discusses. Then, following the standard (first-order) pattern for a de re analysis regarding the referential expression in the sentence said to be believed, Landini suggests that we should move up a level and quantify over that referential expression outside of the context of the nominalized sentence. Thus, the de re analysis he gives us is essentially the following ${ }^{12}$ :

$$
\left.(\exists Q)(\forall G)\left(\left[Q(G) \leftrightarrow\left(\exists_{1} x W\right) G(x)\right] \wedge S \text { believes } Q \text { (Brilliant }\right)\right) .
$$

Note that Landini uses a third-level predicate $Q$ (a la Russell's hierarchy) instead of a variable-binding operator $\mathcal{Q}$ of Frege's hierarchy of unsaturated higher-level concepts (as explained in section 3 of his review). Changing this to an operator gives us the following instead (which I will assume is what Landini really wants):

$$
(\exists \mathcal{Q})(\forall G)\left(\left[(\mathcal{Q} x) G(x) \leftrightarrow\left(\exists_{1} x W\right) G(x)\right] \wedge S \text { believes }(\mathcal{Q} x) \text { Brilliant }(x)\right) .
$$

Now the more natural way of representing the de re analysis of this sentence is simply to bring the referential expression up front as follows:

$$
\left(\exists_{1} x W\right)[S \text { believes Brillisant }(x)] \text {, }
$$

which can be read as ' $S$ believes of the author of Waverly that he is brilliant'. Note that if we assume the extended framework I described in my 1992 paper, we can then obtain

$$
(\exists \mathcal{Q})(\forall G)\left(\left[(\mathcal{Q} x) G(x)=\left[\exists_{1} x W\right) G(x)\right] \wedge S \text { believes }(\mathcal{Q} x) \text { Brilliant }(x)\right],
$$

which is equivalent to (2), and which implies the weaker version (1) above. Formulas (2) and (3), in other words, say essentially the same thing, and (3) implies Landini's (1). Here we have a clear example of how going to a higherlevel is superfluous.

\section{Orilia's Contingent Paradox}

\footnotetext{
${ }^{11}$ In my framework all referential phrases are quantifier phrases, including phrases based on the determiner 'the'. The relevant rule for $\exists_{1}$ is the following schema:

$$
\left(\exists_{1} x A / F(x)\right) G(x) \leftrightarrow(\exists x A)[(\forall y A)(F(y) \leftrightarrow y=x) \wedge G(x)],
$$

I have included the relative clause $F(x)$ here as well for the general case. The quantifier phrase $\left(\exists_{1} x A / F(x)\right)$ is read as 'the $A$ that is $F$ ', and the formula on the left of the biconditional is read as 'the $A$ that is $F$ is $G$ '. The right-hand sign says that there is exactly one $A$ that is $F$ and it is $G$.

${ }^{12}$ Landini has $\left[\lambda H\left(\exists_{1} x W\right) H(x)\right](G)$ instead of $\left(\exists_{1} x W\right) G(x)$, but the two expressions are equivalent by $\lambda$-conversion, and the latter is simpler and easier to read.
} 
Francesco Orilia's contingent paradox starts with specification of a property $P$ defined as follows:

$$
P=\left[\lambda x(\exists F)\left(x=F \wedge(\forall y)\left[S \text { thinks }_{t} \text { about } y \rightarrow \neg F(y)\right]\right)\right] .
$$

Orilia's contingent assumption is that $S$ thinks about an object $y$ at time $t$ if and only if $y$ is $P$ :

$$
(\forall y)\left(S \text { thinks } s_{t} \text { about } y \leftrightarrow y=P\right) .
$$

From this contingent assumption and the definition of $P$, a contradiction is said to follow, namely, $P(P) \longleftrightarrow \neg P(P) \cdot{ }^{13}$

Now this argument may seem familiar to philosophers used to dealing with arguments about propositional attitudes. A familiar example is when the person $S$ is thinking at time $t$ about Cicero. I am not at all sure what is going on in $S$ 's mind when he thinks about Cicero and only Cicero, but according to Landini "we can direct our minds, by employing definite descriptions, to secure reference to a unique object." 14 I find this problematic: can one really "secure reference" to Santa Clause by thinking about Santa Clause whatever definite description one uses? Well, perhaps what Landini means is that we purport to secure reference to Santa Clause by means of a definite description. In any case we'll let this pass and assume that if $S$ thinks at time $t$ about Cicero, then he has a certain definite description in mind, e.g., 'the Roman senator who denounced Cataline'.

But now, although Cicero is Tully, it turns out $S$ does not know this. Yet, it is true that $S$ is thinking at time $t$ about an object $y$ if, and only if $y$ is identical with Cicero, i.e., if, and only if, $y$ is identical with Tully, the Roman senator who denounced Cataline. This is true because the occurrence of 'Cicero' on the right-hand side of the biconditional is referentially transparent, which means that we can replace 'Cicero' with the co-referential name 'Tully' or the definite description 'the Roman senator who denounced Cataline'.

Now it is clear that we cannot validly conclude that $S$ is thinking at time $t$ about Tully - at least not if we want to respect the kind of referential opacity that the variable $y$ has in its occurrence within the scope of the propositional attitude thinking-about. ${ }^{15}$ In other words, because such a context is not referentially transparent, there are restrictions on the use of Leibniz's law when replacing referential expressions that occur within it by co-referential expressions. There are also, I should add, restrictions on the use of universal instantiation into propositional attitude contexts, just as there are such restrictions in modal

\footnotetext{
${ }^{13}$ Orilia 1996.

${ }^{14}$ This view goes back to Russell who wrote that the "names that we commonly use, like 'Socrates', are really abbreviations for descriptions." (Russell 1918, p. 200.)

${ }^{15}$ We might note here that 'think about' is actually doubly opaque, first for 'think' and secondly for 'about'. The intensional preposition can be applied to a number of intensional verbs, such as in 'talk about', 'know about', 'wonder about', etc. The doubly opaque aspect was noted and analyzed by Richard Montague in the type-theoretical intensional logic of his paper, "The proper treatment of quantification in ordinary English," reprinted in Montague 1974.
} 
logic. It would be wrong to ignore these restrictions and conclude that $S$ is both thinking about Tully and not thinking about Tully at time $t$; and it would be inappropriate to claim on the basis of this error that something is wrong, indeed paradoxical, about standard first-order logic with identity.

Applying this familiar way to understand propositional attitude contexts to Orilia's contingent paradox, let us suppose now that what $S$ is thinking about at time $t$ is the property his colleague discussed with him a week before, and that as a matter of fact that property is $P$. Accordingly, Orilia's contingent assumption is true, i.e., $S$ is thinking at time $t$ about an object $y$ if, and only if, $y=P$.

But, although $S$ is thinking at time $t$ about the property his colleague discussed with him a week ago, and this property in fact is $P$, it does not follow that one can validly instantiate the universal quantifier $(\forall y)$ of the contingent assumption to $P$, and conclude that $S$ is thinking at time $t$ about $P$, because in fact what he is thinking about is the property his colleague discussed with him a week ago. Indeed, he may no longer remember the specific structure his colleague had talked about, or he may not even have learned the details of that structure. But even if he did learn and does remember that structure, something more needs to be specified before the universal instantiation in question can be allowed as valid. That is because one of the occurrences of $y$ within the contingent assumption is not referentially transparent, i.e., it does not occur in a strictly extensional context.

Nor similarly can one then go on to instantiate the universal quantifier $(\forall y)$ in the definition of $P$ to $P$, because, as with the contingent assumption, there are restrictions about such a universal instantiation into the context of a propositional attitude. Again, one of the occurrences of $y$ is not referentially transparent; and something more is needed for the universal instantiation to be valid.

Perhaps, in analogy with using Leibniz's law in epistemic contexts, where knowing who $A$ is allows us to treat $A$ as "rigid" in the knower's epistemic space (the possible worlds compatible with what $S$ knows), i.e., where ' $S$ knows who $A$ is' is symbolized as: $(\exists y) S$ knows $[y=A]$, what is needed is something like there being an object $y$ such that $S$ thinks at time $t$ that $y=P$; that is, perhaps the something more that is needed is the following:

$$
(\exists y) S \text { thinks }_{t}[y=P] .
$$

Perhaps this would suffice to stipulate that $P$ is somehow "rigid" in $S$ 's thinking space (whatever that is), so as to indicate that he has learned and hasn't forgotten the involuted details of its specification. But then again, perhaps it does not suffice to account for the something more that is needed.

We do not know without further investigation if this added assumption does suffice for the propositional attitude thinks $s_{t}$, but in any case we are now in a situation where $S$ is thinking at $t$ about more than exactly one thing; that is, he is thinking at time $t$ about the property his colleague discussed with him a week ago, and that some object $y$ is such that he thinks at time $t$ that $y$ is $P$ (whatever that might mean as a mental act). 
The same difficulty applies to Landini's version of the Liar paradox, but I will not into the details here. ${ }^{16}$ The point to keep in mind is that the logic of propositional attitudes, like that of necessity and possibility, requires special constraints on the use of Leibniz's law for identity and for universal instantiation (or existential generalization) - and it requires a semantico-philosophical account of those restrictions as well. What is needed, in other words, is a development of a consistent logic of the expressions for propositional attitudes. That development was not given my book, but was left as a project for future research that I hope both Landini and Orilia, as well as others, will take up in the future.

In any case, as with the so-called paradox of $S$ thinking about Tully and not thinking about Tully, where it would be inappropriate to conclude on the basis of that argument that something is wrong with first-order logic, it would also be inappropriate to conclude on the basis of Orilia's contingent paradox that something is wrong, indeed paradoxical, about the system $\lambda H S T^{*}$ or $H S T_{\lambda}^{*}$, or with my general framework of conceptual realism.

\section{References}

[1] Bozon, Serge, 2004, "Why $\lambda H S T^{*}$ and $H S T_{\lambda}^{*}$ do not Solve the RussellMyhill Paradox After All," Proceedings of the Ninth ESSLLI Student Session, Paul Egré and Lauro Alonso i Alemany, eds., Chapter 1, 2004, p.1.

[2] Church, Alonzo, 1974, "Russellian Simple Type Theory," Proceedings and Addresses of The American Philosophical Association, vol. 47, Dec. 1974: $21-33$.

[3] Church, Alonzo, 1984, "Russell's Theory of Identity of Propositions," Philosophia Naturalis, Band 21, Heft 2-4, 1984: 513-522.

[4] Cocchiarella, Nino B., 1992, "Cantor's Power-Set Theorem Versus Frege's Double-Correlation Thesis," History and Philosophy of Logic, vol. 13 (1992): 179-201.

[5] Cocchiarella, Nino B., 2008, Formal Ontology and Conceptual Realism, Springer, Synthese Library, vol. 339, Dordrecht.

[6] Montague, Richard M., 1974, "The Proper Treatment of Quantification in Ordinary English," reprinted in Formal Philosophy, ed., R.H. Thomason, Yale University Press, New Haven, 1974.

[7] Orilia, Francesco, 1996, "A Contingent Russell Paradox," Notre Dame Journal of Formal Logic 37 (1996): 105-111.

\footnotetext{
${ }^{16}$ The something more that is needed to validate a universal instantiation into a belief context, for example, would lead to a problem with Landini's second assumption for the Liar paradox.
} 
[8] Russell, Bertrand, 1903, Principles of Mathematics, w.w. Norton \& Co., Inc., N.Y., reprinted in 1943.

[9] Russell, Bertrand, 1918, "The Philosophy of Logical Atomism", reprinted in Logic and Knowledge, ed., Robert C. Marsh, George Allen and Unwin LTD, London. 